\title{
Editorials
}

\section{Science, pseudoscience and Sellick}

\author{
J. Roger Maltby MB BCHIR FRCA FRCPC, Michael T. Beriault MD FRCPC
}

$\mathrm{P}$ ULMONARY aspiration of gastric contents remains a much-feared complication of anesthesia. Anesthesiologists commonly refer to patients as being at 'high risk' of pulmonary aspiration. Risk means that an unpleasant or dangerous outcome may occur. The frequency of that outcome quantifies the risk. We accept assumptions about 'risk factors' that will reduce or increase that frequency, while giving little attention to the evidence. We take steps to prevent aspiration and are satisfied that our knowledge and technical expertise appear to be effective. Management strategies are based on those assumptions but, if the assumptions are not evidencebased, logical deductions may lead to invalid or erroneous conclusions. We have made pilgrimages to many shrines in the past 40 years, always in search of the amulet that will ward off aspiration, and the demons of blame and litigation. How 'high' is the 'high risk' that we fear? Epidemiological evidence suggests that because our fear of aspiration is exaggerated, all strategies appear to be effective.

In 1946, Mendelson ${ }^{1}$ published his retrospective review of anesthetic related morbidity and mortality in more than 44,000 pregnancies from 1932 to 1945 at the Lying-In Hospital in New York. He described the typical clinical and radiological changes following liquid aspiration. All anesthetics in these cases were nitrous oxide and ether, without tracheal intubation, and were often given by inexperienced interns. There were 66 cases of aspiration. No deaths occurred in the 40 who aspirated liquid, but two of five patients who aspirated solids succumbed. No deaths occurred in those cases in which the aspirated material was not recorded.

In 1986, Olsson ${ }^{2}$ reported 83 aspirations with four deaths in 185,358 anesthetics $(1: 45,454)$. Two of the four were already very ill, one had a failed intubation, and one vomited under spinal anesthesia. In 1993, Warner $^{3}$ reported three deaths, all in ASA III-V patients, in 215,488 general anesthetics $(1: 71,829)$. He defined aspiration as bilious secretions or particulate matter in the tracheobronchial tree or a new infiltrate on postoperative chest $x$-ray. The incidence varied from 1:9,229 for ASA I patients undergoing elective surgery to 1:895 for all emergency surgery. Obstetric patients were the only ones who received routine antacids, $\mathrm{H}_{2}$ receptor blockers and gastrokinetic medications. More than half (18 of 29) of those who had specific risk factors and aspirated had received pharmacological prophylaxis. The most consistent contributing factors were gagging and vomiting during laryngoscopy (33\%) or gagging and vomiting during emergence from anesthesia (36\%).

Vomiting is the forcible expulsion of gastric contents into the pharynx as the lower and upper esophageal sphincters relax. It requires skeletal muscle activity. Regurgitation is a passive process. Recommended anesthetic techniques for the 'full stomach' patient in the $1950 \mathrm{~s}$, before the days of succinylcholine, halothane and cricoid pressure, now sound bizarre. ${ }^{4}$ Nevertheless, they were based on physiological principles and appeared to prevent pulmonary aspiration. The Newcastle technique was based on the premise that a patient cannot hyperventilate and vomit at the same time. An inhalation induction of nitrous oxide, oxygen, carbon dioxide and ether was used to produce hyperventilation until anesthesia was deep enough for tracheal intubation. Other techniques involved a 20 head-down tilt, or head-down tilt in the lateral position that assisted drainage of fluid away from the glottis, but these made laryngoscopy difficult. The $40^{\circ}$ head-up tilt position raised the larynx $19 \mathrm{~cm}$ above the lower esophageal sphincter. ${ }^{4}$ This strategy was based on O'Mullane's clinical studies, ${ }^{5}$ which showed that, even with 500-1,000 $\mathrm{mL}$ saline in the stomach, the intragastric pressure did not exceed $18 \mathrm{~cm}$ water. The head-up tilt would therefore prevent passive reflux into the phar-

From the Department of Anesthesia, Foothills Medical Centre, Calgary, Alberta, Canada.

Address correspondence to: Dr. J. Roger Maltby, Department of Anesthesia, Foothills Medical Centre, 1403 - 29th Street N.W., Calgary, Alberta T2N 2T9, Canada. Phone: 403-670-1667; Fax: 403-670-2425; E-mail: maltby@ucalgary.ca 
ynx, but would unfortunately increase the likelihood of aspiration if active vomiting occurred.

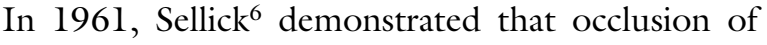
the esophagus by cricoid pressure in cadavers prevented flow of barium from the stomach to the pharynx. He reported successful use of this technique in 26 'full stomach' cases - intestinal obstruction, gastrectomy for pyloric stenosis, and forceps delivery. In three cases, the pharynx filled with gastric contents when cricoid pressure was released after inflation of the tracheal tube cuff. He advocated passage of a gastric tube to empty the stomach. He described, with illustrations, precisely how cricoid pressure should be applied. Sellick's maneuver changed the management of anesthesia worldwide.

In 1974, Roberts and Shirley suggested that a gastric fluid volume of $0.4 \mathrm{~mL} \cdot \mathrm{kg}^{-1}$ or $25 \mathrm{~mL}$ in the adult stomach put patients at 'high risk' for pulmonary aspiration. ${ }^{7}$ In 1980, they revealed that this was based on a single experiment in one monkey. The liquid was not regurgitated from its stomach, but was injected into the right main bronchus from a syringe. Meanwhile, clinical studies demonstrated that the gastric fluid volume in $40-80 \%$ of healthy, fasting patients exceeded the surrogate marker of $25 \mathrm{~mL}$ with $\mathrm{pH}<2.5 .^{8}$ The logical response to these surrogate endpoints was to modify gastric contents pharmacologically. More than a decade later, Olsson ${ }^{2}$ and Warner ${ }^{3}$ showed that fear of pulmonary aspiration was grossly exaggerated. Twentyseven years after Roberts and Shirley, there are still no data that show improved outcome with $\mathrm{H}_{2}$ receptor blockers, gastrokinetics or foul-tasting sodium citrate. ${ }^{9}$

For many years, 'NPO after midnight' orders were applied to clear liquids as well as to solids to ensure an 'empty stomach.' Randomized studies have shown that drinking clear liquids on the day of surgery does not increase gastric fluid volume. The American Society of Anesthesiologists guidelines now recommend that healthy patients for elective surgery may drink clear liquids until two hours before surgery, and that routine pharmacological prophylaxis is not indicated. ${ }^{10}$

In this issue, Smith et al. ${ }^{11}$ report a retrospective review of 51 cervical computed tomography scans of normal patients. They measured esophageal diameter, distance between the midline of the cricoid and the midline of the esophagus, and distance between the lateral border of the cricoid and the lateral border of the esophagus. The esophagus was displaced laterally in half $(25 / 51)$ of the patients. Displacement extended beyond the lateral border of the cricoid in one third $(16 / 51)$. Is it possible that this finding will explain the occasional failure of cricoid pressure to prevent gastric contents from reaching the pharynx and lungs?
The answer is probably that we shall never know, but the finding should give the reader a sense of déjà vu. The incidence discrepancy between this new surrogate risk marker and clinical outcome of pulmonary aspiration is strikingly similar to that for 'high risk' gastric contents. ${ }^{12}$ We do not question the internal validation of Smith's paper. However, external validation is unlikely to be forthcoming until the study is repeated with the patient's head in the intubating position. Sellick ${ }^{6}$ was specific in his description of this position when he applied cricoid pressure: "The head and neck are fully extended (as in the position for tonsillectomy). This increases the anterior convexity of the cervical spine, stretches the esophagus, and prevents its lateral displacement when pressure is applied to the cricoid."

Although cricoid pressure appears to be a simple mechanical maneuver that prevents passage of gastric contents into the pharynx, no randomized controlled trial has ever been conducted to assess its effectiveness. Cricoid pressure has become the unofficial standard practice in patients who are believed to be at high risk' of pulmonary aspiration, but there is no substantial agreement on exactly who those patients are. A survey of one Canadian university centre showed an overall use in $19 \%$ of 3,961 cases, with a range of $5-34 \%$ among nine anesthesiologists. ${ }^{13}$

Much of the recent cricoid pressure literature focuses more on required force than proven clinical benefit. Cricoid pressure increases upper esophageal sphincter pressure and may prevent regurgitation and gastric insufflation. It also decreases lower esophageal sphincter pressure, impedes correct placement of the laryngeal mask airway (LMA), and tracheal intubation through the intubating LMA. ${ }^{14}$ The force that is actually applied is highly subjective. In a study of anesthetic assistants, it ranged from $<30 \mathrm{~N}$ to $>90 \mathrm{~N}$. Forces $>40 \mathrm{~N}$ may cause airway obstruction, difficult intubation and even esophageal rupture. A force of $30 \mathrm{~N}$ is sufficient to prevent regurgitation of esophageal fluid with a pressure of $40 \mathrm{~mm}$ in cadavers. ${ }^{15}$ This is more than double the normal gastric pressure of $10-15 \mathrm{mmHg}$.

Backward and upward force on the larynx improves laryngoscopic view, but should be backward only if cricoid pressure is required. If manual ventilation is required, or it may have to be released entirely. These disadvantages may make airway management more difficult and therefore more dangerous if it is used indiscriminately in patients at low risk of aspiration. If cricoid pressure makes laryngoscopy difficult, and manipulation of the airway under light anesthesia provokes gagging and vomiting, its indiscriminate use may cause real 
problems while trying to solve hypothetical ones. It should be reserved for cases at genuine higher risk, like those mentioned by Sellick in his study.

\section{Science, pseudoscience et Sellick}

L'aspiration pulmonaire du contenu gastrique demeure une hantise en anesthésie. Les anesthésiologistes considèrent habituellement que les patients sont à "haut risque" d'aspiration pulmonaire. Le risque étant ici qu’un événement désagréable ou dangereux puisse survenir. La fréquence de cet événement quantifie le risque. Nous acceptons l'idée que "des facteurs de risque" puissent influencer cette fréquence, tout en prêtant peu d'attention aux données probantes. Nous prenons des mesures pour prévenir l'aspiration et sommes heureux que nos connaissances et notre savoir-faire technique semblent porter leurs fruits. Nos stratégies d'intervention sont fondées sur ces hypothèses de base, mais si les hypothèses ne sont pas prouvées, des déductions logiques peuvent mener à des conclusions invalides ou erronées. Pendant les 40 dernières années, nous avons fait de nombreuses recherches comme autant de pèlerinages dans différents sanctuaires pour trouver une amulette qui allait nous libérer des problèmes d'aspiration, et repousser les démons du blâme et des poursuites. Mais quelle est, en fait, la "hauteur" de ce "haut risque" que nous craignons ? La preuve épidémiologique permet de dire que notre crainte de l'aspiration est exagérée, de sorte que toutes les stratégies paraissent efficaces.

En 1946, Mendelson ${ }^{1}$ a publié sa revue rétrospective de la morbidité et de la mortalité reliées à l'anesthésie pour plus de 44000 grossesse entre 1932 et 1945 au Lying-In Hospital de New York. Il a décrit les changements cliniques et radiologiques caractéristiques qui suivent l'aspiration de liquide. Les anesthésiques étaient du protoxyde d'azote et de l'éther, utilisés sans intubation, et souvent par des internes inexpérimentés. Il y a eu 66 cas d'aspiration. Aucun décès n'est relié aux 40 cas d'aspiration de liquide, mais deux des cinq patients qui avaient aspiré des solides sont décédés. Aucun décès n'est lié aux cas où les matières aspirées n'ont pas été notées.

En 1986, Olsson ${ }^{2}$ a rapporté 83 aspirations, dont quatre suivies de décès, pour 185358 anesthésiques (1 : 45 454). Deux des patients décédés étaient très malades, l'un n'a pu être intubé et l'autre a vomi pendant la rachianesthésie. En 1993, Warner ${ }^{3}$ a signalé trois décès de patients d'état physique ASA III-V, pour 215488 anesthésiques généraux (1 : 71 829). L'aspiration consistait en sécrétions bilieuses ou en particules dans l'arbre trachéo-bronchique ou en un nouvel infiltrat révélé par la radiographie postopératoire. L'incidence variait de $1: 9229$ pour les patients ASA I de chirurgie élective à $1: 895$ pour toutes les opérations d'urgence. Seules les patientes d'obstétrique avaient reçu des antiacides de routine, des bloqueurs des récepteurs $\mathrm{H}_{2}$ et des traitements pour accélérer la vidange gastrique. Plus de la moitié de celles qui présentaient des facteurs de risque spécifique et avaient eu des problèmes d'aspiration avaient reçu une thérapie pharmacologique préventive. Les facteurs en jeu étaient surtout les hauts-le-cœur et les vomissements pendant la laryngoscopie $(33 \%)$ ou pendant le retour à la conscience après l'anesthésie (36\%).

Le vomissement est l'expulsion forcée du contenu gastrique dans le pharynx associé à un relâchement des sphincters œsophagiens inférieur et supérieur. Il nécessite l'activité des muscles squelettiques. La régurgitation est un processus passif. Les techniques anesthésiques recommandées dans les années 1950 dans les cas “d'estomac plein", avant l'arrivée de la succinylcholine, de l'halothane et de la compression cricoïdienne, nous semblent maintenant étranges. ${ }^{4}$ Elles étaient néanmoins fondées sur des principes physiologiques et ont semblé prévenir l'aspiration pulmonaire. Le fondement de la technique Newcastle était que l'hyperventilation et les vomissements ne peuvent être simultanés chez un même patient. Une induction par inhalation de protoxyde d'azote, d'oxygène, de gaz carbonique et d'éther a été utilisée pour produire une hyperventilation jusqu'à ce que l'anesthésie soit assez profonde pour permettre l'intubation endotrachéale. On a aussi expérimenté une position de Trendelenburg à $20^{\circ}$ ou Trendelenburg en décubitus latéral pour faciliter le drainage du liquide hors de la glotte, ce qui complique la laryngoscopie, et une position de Fowler à $40^{\circ}$, ce qui élève le larynx de $19 \mathrm{~cm}$ au-dessus du sphincter inférieur de l'œsophage. ${ }^{4}$ Cette position a été adoptée à la suite des études cliniques de O'Mullane ${ }^{5}$ qui ont montré que, même avec $500-1000 \mathrm{~mL}$ de solution saline dans l'estomac, la pression intragastrique ne dépasse pas 18 $\mathrm{cm}$ d'eau. La tête vers le haut peut donc prévenir le reflux passif dans le pharynx, mais accroître la probabilité d'aspiration en cas de vomissement.

En 1961, Sellick ${ }^{6}$ a démontré que, chez les cadavres, l'occlusion de l'œsophage par la pression sur le cricoïde empêche l'écoulement de baryum de 
l'estomac vers le pharynx. Il a rapporté l'utilisation réussie de cette technique dans 26 cas "d'estomac plein" - pendant des opérations pour obstruction intestinale, gastrectomie pour sténose du pylore, accouchement avec forceps. Dans trois cas, le contenu gastrique a envahi le pharynx lorsque la compression cricoïdienne a été relâchée après le gonflement du ballonnet du tube endotrachéal. Sellick a suggéré l'usage d'un tube gastrique pour vider l'estomac. Il a décrit, et illustré précisément, comment appliquer la pression sur le cricoïde. La manœuvre de Sellick a transformé l'anesthésie à travers le monde.

En 1974, Roberts et Shirley ont avancé que, chez un adulte, la présence de $0,4 \mathrm{~mL} \cdot \mathrm{kg}^{-1}$ ou de $25 \mathrm{~mL}$ de liquide gastrique constituait un risque élevé d'aspiration pulmonaire. ${ }^{7}$ En 1980, ils ont avoué que ce résultat faisait suite à une seule expérience avec un singe. Le liquide n'avait pas été régurgité de l'estomac, mais injecté dans la bronche principale droite avec une seringue. Entre-temps, des études cliniques ont démontré que le volume de liquide gastrique chez 40 à $80 \%$ des patients en bonne santé, à jeun, dépasse le niveau cible de $25 \mathrm{~mL}$ avec un $\mathrm{pH}<2,5 .{ }^{8}$ La réponse logique à ces paramètres substituts a été de modifier le contenu gastrique de manière pharmacologique. Plus d'une décennie après, Olsson ${ }^{2}$ et Warner ${ }^{3}$ ont montré que la crainte d'une aspiration pulmonaire était nettement exagérée. Il n'y a toujours pas, 27 ans après Roberts et Shirley, de meilleurs résultats obtenus avec les bloqueurs de récepteurs $\mathrm{H}_{2}$, les gastrocinétiques ou le citrate de sodium au goût infect. ${ }^{9}$

Pendant de nombreuses années, la consigne "NPO après minuit" a été appliquée pour éliminer les liquides et les solides et garantir un "estomac vide". Des études randomisées ont montré que boire des liquides clairs le jour d'une opération n'augmente pas le volume de liquide gastrique. L'American Society of Anesthesiologists est maintenant d'avis que les sujets en bonne santé admis en chirurgie non urgente peuvent boire des liquides clairs jusqu'à deux heures avant l'opération et qu'une prophylaxie pharmacologique de routine n'est pas indiquée. ${ }^{10}$

Dans le présent numéro, Smith et coll. ${ }^{11}$ présentent une revue rétrospective de 51 examens tomodensitométriques cervicaux de patients normaux. Ils ont mesuré le diamètre de l'osophage, la distance entre les lignes médianes du cricoïde et de l'œsophage et la distance entre le bord latéral du cricoïde et celui de l'œsophage. L'œsophage était déplacé latéralement chez la moitié $(25 / 51)$ des patients. Le déplacement s'étendait au-delà du bord latéral du cricoïde dans un tiers des cas (16/51). Est-il possible que ce résultat explique pourquoi la compression cricoïdienne ne réussit pas, à l'occasion, à empêcher le contenu gastrique d'atteindre le pharynx et les poumons?

Nous ne le saurons peut-être jamais. La situation devrait toutefois laisser au lecteur une impression de déjà vu. La différence d'incidence entre ce nouveau marqueur de risque substitut et l'aspiration pulmonaire constatée en clinique est étonnamment similaire à celle qu'on a établie avec les contenus gastriques à "haut risque". ${ }^{12}$ Nous ne remettons pas en cause la validité interne de l'article de Smith. Cependant, la validité externe ne pourra être établie qu'en répétant l'étude et en plaçant la tête du patient en position d'intubation. Sellick ${ }^{6}$ décrit spécifiquement cette position : "La tête et le cou sont en extension complète (comme la position adoptée pour l'amygdalectomie). Cette position augmente la convexité antérieure de la colonne cervicale, étire l' $\alpha$ sophage et prévient son déplacement latéral lorsque la pression est appliquée sur le cricoïde."

La compression cricoïdienne semble une simple manœuvre mécanique qui empêche le passage du contenu gastrique dans le pharynx, mais son efficacité n'a fait l'objet d'aucun essai randomisé et contrôlé. Elle est devenue une norme de pratique non officielle pour les patients jugés à "haut risque" d'aspiration pulmonaire, mais on ne s'entend pas sur la définition exacte de ces patients. Une enquête auprès de neuf anesthésiologistes d'un centre universitaire canadien a montré son utilisation dans $19 \%$ de 3961 cas, variant entre $5-34 \%$ selon l'anesthésiologiste. ${ }^{13}$

Dans la plupart des articles récents concernant la compression cricoïdienne, on insiste davantage sur la force nécessaire à son application plutôt que sur ses bénéfices cliniques éprouvés. Cette compression augmente la pression du sphincter œsophagien supérieur et peut empêcher la régurgitation et l'insufflation gastrique. De plus, elle diminue la pression du sphincter œsophagien inférieur, gêne la bonne mise en place du masque laryngé (ML) et l'intubation à travers le masque laryngé d'intubation. ${ }^{14} \mathrm{La}$ force réellement appliquée est très subjective. Dans une étude auprès d'assistants anesthésistes, les mesures allaient de < $30 \mathrm{~N}$ à $>90 \mathrm{~N}$. Des forces $>40 \mathrm{~N}$ peuvent entraîner une obstruction des voies aériennes, une intubation difficile et même la rupture de l'œsophage. Chez des cadavres, une force de $30 \mathrm{~N}$ suffit à prévenir la régurgitation du liquide œsophagien maintenue à une pression de $40 \mathrm{~mm} .{ }^{15}$ C'est plus du double de la pression gastrique normale de $10-15 \mathrm{mmHg}$.

La poussée postérieure et ascendante sur le cricoïde améliore la visualisation laryngoscopique, mais le mouvement vers l'arrière doit être utilisé seulement si la ventilation manuelle est nécessaire. Autrement, c'est 
possible qu'on doive la relâcher complètement. L'assistance respiratoire peut devenir alors plus difficile, voire plus dangereuse, si la compression cricoïdienne est utilisée sans distinction chez des patients à faible risque d'aspiration. Sachant que la compression cricoïdienne complique la laryngoscopie, et que la manipulation des voies aériennes sous anesthésie légère provoque des hauts-le-cœur et des vomissements, on ne peut l'utiliser sans distinction et risquer de causer de réels problèmes en voulant en résoudre d'autres, hypothétiques. La technique doit être réservée aux cas vraiment à risque très élevé, comme ceux de l'étude de Sellick.

\section{References}

1 Mendelson CL. The aspiration of stomach contents into the lungs during obstetric anesthesia. Am J Obst Gynecol 1946; 52: 191-205.

2 Olsson GL, Hallen B, Hambraens-Jonzon K. Aspiration during anaesthesia: a computer-aided study of 185358 anaesthetics. Acta Anaesthesiol Scand 1986; 30: 84-92.

3 Warner MA, Warner ME, Weber JG. Clinical significance of pulmonary aspiration during the perioperative period. Anesthesiology 1993; 78: 56-62.

4 Inkster JS. The induction of anaesthesia in patients likely to vomit with special reference to intestinal obstruction. Br J Anaesth 1963; 35: 160-7.

5 O'Mullane EJ. Vomiting and regurgitation during anaesthesia. Lancet 1954; 1: 1209-12.

6 Sellick BA. Cricoid pressure to control regurgitation of stomach contents during induction of anaesthesia.

Lancet 1961; 2: 404-6.

7 Roberts RB, Shirley MA. Reducing the risk of acid aspiration during cesarean section. Anesth Analg 1974; 53: 859-68.

8 Moyers JR, Vincent CM. Preoperative medication. In: Barash PG, Cullen BF, Stoelting RK (Eds.). Clinical Anesthesia, 4th ed. Philadelphia: Lippincott-Williams \& Wilkins, 2001: 551-65.

9 Engelhardt T, Webster NR. Pulmonary aspiration of gastric contents in anaesthesia. Br J Anaesth 1999; 83: 453-60.

10 American Society of Anesthesiologists Task Force on Preoperative Fasting. Practice guidelines for preoperative fasting and the use of pharmacologic agents to reduce the risk of pulmonary aspiration: application to healthy patients undergoing elective procedures. A report by the American Society of Anesthesiologists Task Force on Preoperative Fasting. Anesthesiology 1999; 90: 896-905.

11 Smith KJ, Ladak S, Choi PT-L, Dobranowski J. The cricoid cartilage and the esophagus are not aligned in close to half of adult patients. Can J Anesth 2002; 49: 503-07.
12 Schreiner MS. Gastric fluid volume: is it really a risk factor for pulmonary aspiration? Anesth Analg 1998; 87: 754-6.

13 Edington RD, King FG, LeDez KM. Utilization of cricoid pressure in a teaching hospital. Can J Anesth 1999; 46: All (abstract).

14 Brimacombe J, Berry AM. Cricoid pressure. Can J Anaesth 1997; 44: 414-25.

15 Vanner RG. Cricoid pressure in chaos (Letter). Anaesthesia 1998; 53: 94. 